\title{
In-plane illuminated metallic annular aperture array for sensing application
}

\author{
Qing Tan,* Matthieu Roussey, Armando Cosentino, and Hans Peter Herzig \\ Ecole Polytechnique Fédérale de Lausanne, Institute of Microengineering, Optics \& Photonics Technology Laboratory \\ Breguet 2, 2000 Neuchâtel, Switzerland \\ *Corresponding author: qing.tan@epfl.ch
}

Received October 17, 2011; revised November 30, 2011; accepted December 29, 2011; posted January 3, 2012 (Doc. ID 156372); published February 10, 2012

\begin{abstract}
We propose here an application to sensing of annular aperture arrays (AAA). We theoretically investigate the optical properties of the reflective AAA device when illuminated in-plane. The cavity presents almost perfect absorption due to the waveguide mode resonance with strong field localization in the aperture. Additionally, the reflective cavity is modeled to be available for on-chip sensing with a theoretically expected sensitivity of $764 \mathrm{~nm} / \mathrm{RIU}$ (refractive index unit). (c) 2012 Optical Society of America

OCIS codes: $\quad 050.6624,130.6010,310.6628,050.1755$.
\end{abstract}

During the last decade, it has been demonstrated $[\underline{1}, \underline{2}]$ that a thin nanopatterned metallic film may exhibit an unexpected enhanced transmission compared to Bethe's theory [3] under particular conditions. Several theories $[\underline{4}, \underline{5}]$ and experiments $[\underline{6}-\underline{8}]$ have been carried out to understand and optimize this phenomenon. The origin of this extraordinary transmission changes depending on the shape of the apertures. Two principal categories exist: circular aperture arrays and annular aperture arrays (AAA). In the first case, the transmission is due to surface plasmon coupling on both sides of the thin film [6]. In the second case, the phenomenon comes from the existence of a guided mode inside the cavity. With regard to applications, this geometry [9] is applicable to on-chip sensing and promises high sensitivity to environmental property variations. Recently, biosensors based on the transmission spectrum of nanohole arrays were developed in combination with nanofluidics $[\underline{10}, 11]$. Furthermore, disk or ring resonators have shown promising results in terms of sensitivity [around $600 \mathrm{~nm} / \mathrm{RIU}$ (refractive index unit)], with the advantage of planar integration on chips $[12,13]$. Since a high field localization has been demonstrated in the narrow dielectric region of the metal/ dielectric/metal aperture [14] and thanks to our recent result obtained with slot waveguide cavity [15], we propose here to investigate the behavior of the electromagnetic field inside coaxial apertures when illuminated in-plane. It appears as a combination of high transmission and planar optics with strong field localization, which should provide a high sensitivity associated to a local interaction with the measured medium. In this Letter, the integration of these nanostructures is considered for embedment in waveguides.

Figure 1 presents the schematic view of three periods of the studied structure. It consists of one row of annular holes in a block of gold $(\mathrm{Au})$ embedded on a silicon nitride $\left(\mathrm{Si}_{3} \mathrm{~N}_{4}\right)$ waveguide. The different parameters are: the periodicity $(p)$, the cut-distance $\left(d_{0}\right)$, the inner radius $\left(r_{i}\right)$, the outer radius $\left(r_{e}\right)$ of the circular aperture, and the block metal thickness $\left(d_{1}\right)$. The incident medium $\left(\mathrm{Si}_{3} \mathrm{~N}_{4}\right)$ has a refractive index of $n_{1}=1.98$, while water is first considered to fill the aperture of the Au cavity with a refractive index of $n_{2}=1.32$. The dispersion property of $\mathrm{Au}$ is modeled by a well-fitted Drude model around the working wavelength $1.55 \mu \mathrm{m}$. TM polarized light is normally injected to the cavity with the electric field component perpendicular to the axis of the annular apertures.

The device is intended to be a gas or liquid sensor based on refractive index variation measurements. To obtain a high sensitivity, the confinement of light in the cavity has to be optimized in order to increase the interaction between light and analyte. Figure 2 presents finite difference time domain (FDTD) modeled reflection spectra for period variations. One can observe a principal resonance shift to the red when the period increases. A minimal reflection (close to zero) is observed for $p \simeq$ $600 \mathrm{~nm}$ at $\lambda=1.53 \mu \mathrm{m}$. For our application, only this period will be considered. Indeed, a low reflection corresponds to a high absorption of light inside the cavity where the analyte will be placed.

Figure 3 presents the phase profile of the field along the circular aperture (dashed line $S_{1}$ ) and through the cavity along the central line (dashed line $S_{2}$ ) in Fig. 1 . Figure 3(a) shows a phase step of $\pi$. Light is separated in two equal parts at the entrance of the cavity. One wave is propagating clockwise and another is propagating counter clockwise, which creates a standing wave in the annular aperture. Figure 3(b) shows a propagating wave before and after the cavity region, which proves that a part of the light can pass through the block of gold

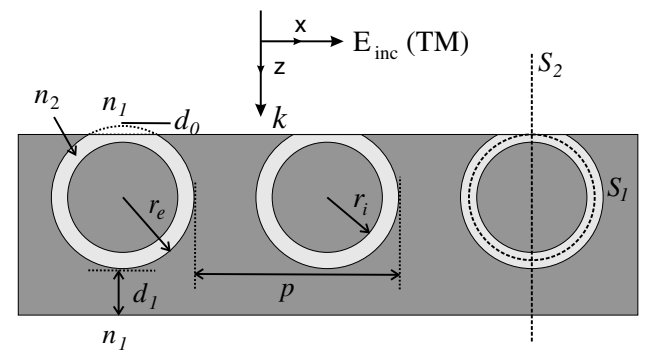

Fig. 1. Schematic view of the studied cavity. TM polarized light is normally incident on the periodic cavity. 

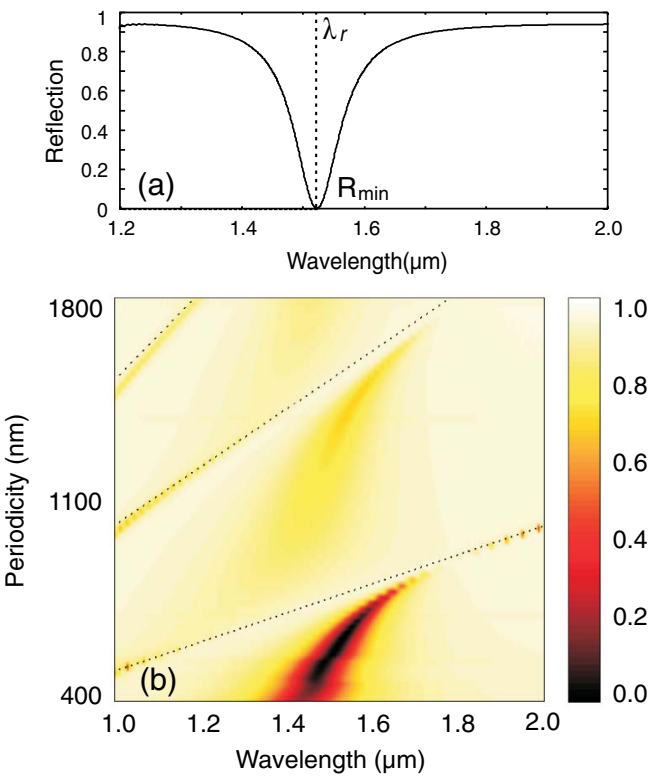

Fig. 2. (Color online) Calculated reflection spectra for (a) $p=600 \mathrm{~nm}$, (b) varying periods (400 nm $<p<1800 \mathrm{~nm}$ ). Other cavity parameters are $r_{e}=180 \mathrm{~nm}, \quad r_{i}=140 \mathrm{~nm}$, $d_{0}=10 \mathrm{~nm}$. The dotted lines present Wood's anomaly conditions $\lambda=p \times n_{\mathrm{Si}_{3} \mathrm{~N}_{4}} / m$ with $m=1,2,3$, respectively.

(transmission smaller than 1\%) and one observes a phase shift of $\pi$ between the input and the output of the cavity, which corresponds to the first mode of a Fabry-Pérot interferometer (Fizeau interferometer). This behavior allows a high confinement of the electromagnetic field in the annular aperture.

The shape, the amplitude, and the spectral position of the guided mode resonance depends on several parameters. In first approximation, we can compare the structure to a Fabry-Pérot to identify the parameters. Using this hypothesis, it is clear that the effective index of the mode excited inside the cavity and the length of the cavity are crucial. In our case, the effective index depends on
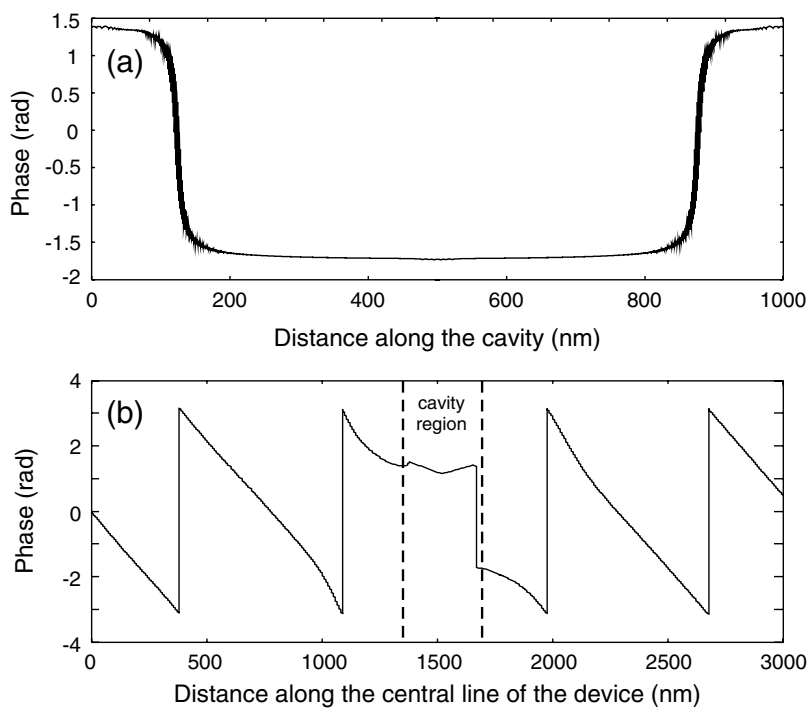

Fig. 3. Phase profile (a) along the cavity (dashed line $S_{1}$ in Fig. 1) and (b) through the cavity along the central line (dashed line $\bar{S}_{2}$ in Fig. 1) at $\lambda_{r}=1530 \mathrm{~nm}$.
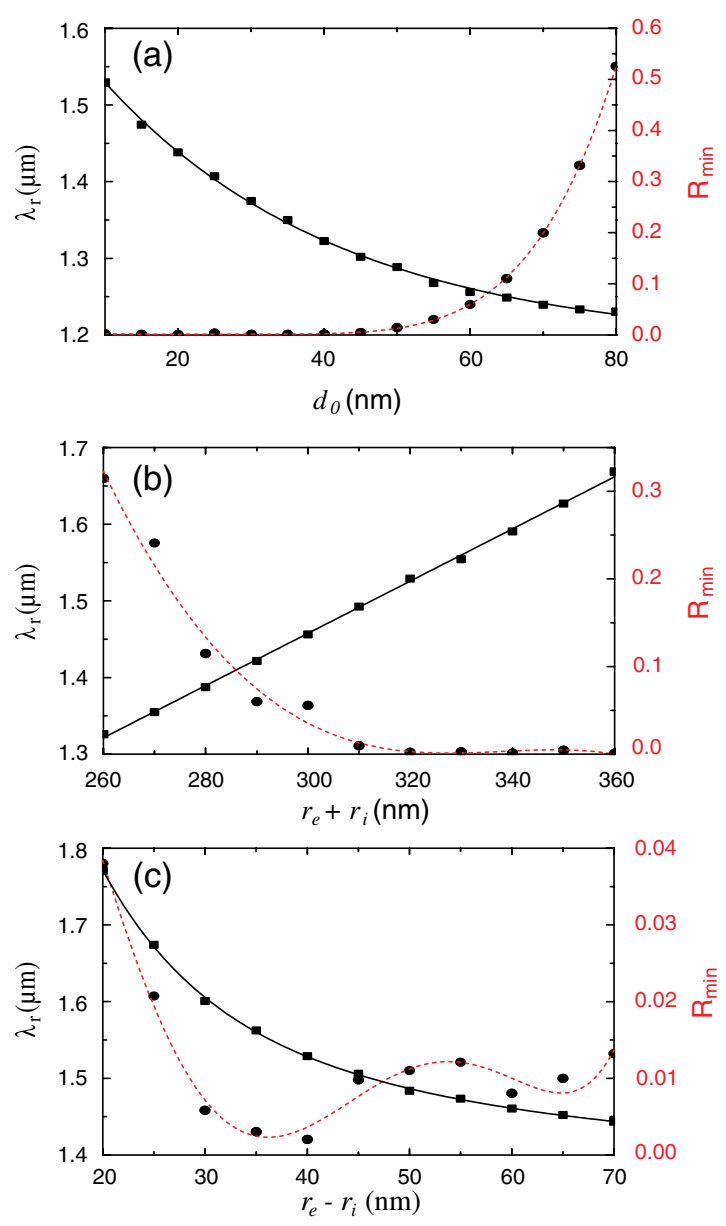

Fig. 4. (Color online) Representation of the position of the dip $\lambda_{r}$ (solid curve) and the minimal reflection $R_{\min }$ (dashed curve) in function of (a) the cut-distance $d_{0}$, (b) the radii sum $r_{e}+r_{i}$ and (c) the radii difference $r_{e}-r_{i}$.

the width of the coaxial aperture (radii difference $\left.r_{e}-r_{i}\right)$, the cut-distance $d_{0}$, and the analyte refractive in$\operatorname{dex} n_{2}$. In order to observe the influence of each geometrical parameter we have performed FDTD calculations summarized in Fig. 4. In Fig. 4(a) we vary the cut-distance $\left(d_{0}\right)$, which may drastically change the response of the structure. Indeed, it corresponds to the opening of the aperture and also influences the length of the cavity. As it is shown in Fig. 4(a), the reflection is close to 0 when $d_{0}<\left(r_{e}-r_{i}\right)$. After this limit, the reflection

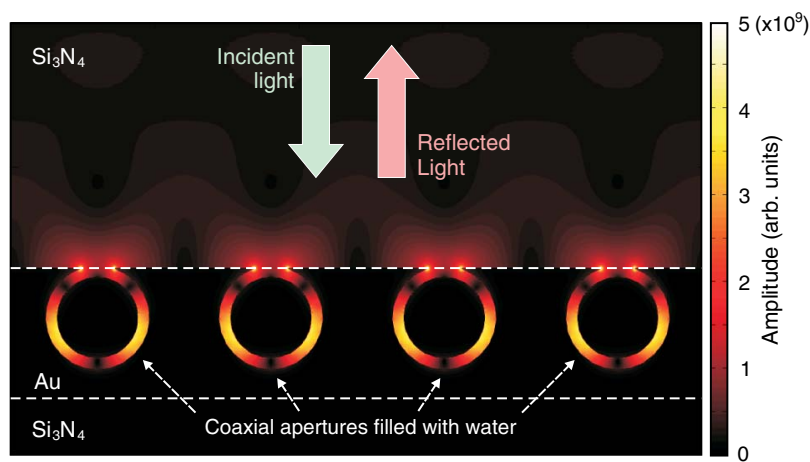

Fig. 5. (Color online) Distribution of the electric field amplitude in the structure at the resonance $\left(\lambda_{r}=1530 \mathrm{~nm}\right)$. 


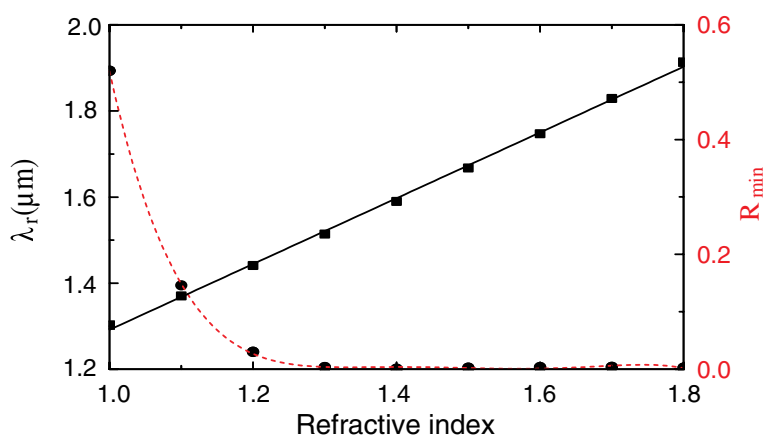

Fig. 6. (Color online) Resonance position $\lambda_{r}$ (solid line) and minimal reflection $R_{\min }$ (dashed line) at the position in function of the refractive index of the medium inside and above the cavity.

increases. The radii sum is proportional to the length of the cavity. The position of the dip depends linearly with the radii sum, which is expected if the cavity acts as a Fabry-Pérot: the resonance wavelength almost linearly increases with the length of the cavity under the condition of weakly dispersive property of the excited mode (not shown here). Nevertheless, for small radii sum, the reflection remains high and the coupling in the annular aperture is not efficient. The radii difference corresponds to the width of the cavity. The width of cavity does not affect strongly the reflectivity $\left(R_{\min }<0.04\right)$ in the graph and $\lambda_{r}$ increases when $r_{e}-r_{i}$ decreases. The study of these parameters allows us to determine the ideal parameters to obtain the best coupling of light in the cavity. Figure 5 represents the electric field amplitude distribution $(|\bar{E}|)$ in the structure at the dip wavelength. The electromagnetic field is highly confined in the aperture, especially at the entrance of the cavity where we can observe a strong lightning rod effect. In such a case the maximum light will interact with the analyte and it corresponds to the maximal sensitivity achievable.

The sensing principle is to place the liquid or the gas to analyze on top of the nanostructure. The analyte will then fill the empty region of the cavity. When the refractive index above and inside the device is modified, the reflection dip is shifted from its original position. Figure $\underline{6}$ shows a linear response of the sensor on a large range of refractive index values with a sensitivity $\eta=\frac{\Delta \lambda}{\Delta n} \simeq$ $764 \mathrm{~nm} / \mathrm{RIU}$. One can remark that for gases, which have refractive indices close to $n=1$, the contrast is lower since $R_{\min } \gg 0$. For liquids and elements in water solution (such as biological media), with an index around $n=1.3$ the contrast will be maximal. In this Letter we have studied the potential application to sensing of subwavelength coaxial apertures embedded in a $\mathrm{Si}_{3} \mathrm{~N}_{4}$ waveguide. In particular, we have highlighted the Fabry-Pérot like behavior and its flexibility in the parameter design. The existence of a guided mode allows a high confinement in the cavity and especially field localization at the entrance of the cavity. As a consequence, a high sensitivity to the refractive index variation is theoretically expected for a large panel of analytes. Moreover, the field at the entrance of the aperture can be further enhanced and is interesting for the development of sample detection with a tiny volume.

The authors wish to thank the Swiss National Science Foundation (SNF) and the European Space Agency (ESA) for their financial support.

\section{References}

1. T. W. Ebbesen, H. J. Lezec, H. F. Ghaemi, T. Thio, and P. A. Wolff, Nature 391, 667 (1998).

2. F. I. Baida and D. Van Labeke, Opt. Commun. 209, 17 (2002).

3. H. A. Bethe, Phys. Rev. 66, 163 (1944).

4. L. Martin-Moreno, F. J. Garcia-Vidal, H. J. Lezec, K. M. Pellerin, T. Thio, J. B. Pendry, and T. W. Ebbesen, Phys. Rev. Lett. 86, 1114 (2001).

5. F. I. Baida, D. Van Labeke, G. Granet, A. Moreau, and A. Belkhir, Appl. Phys. B 79, 1 (2004).

6. C. Genet and T. W. Ebbesen, Nature 445, 39 (2007).

7. Y. Poujet, M. Roussey, J. Salvi, F. I. Baida, D. Van Labeke, A. Perentes, C. Santschi, and P. Hoffmann, Photon. Nanostruct. Fundam. Applic. 4, 47 (2006).

8. Y. Poujet, J. Salvi, and F. I. Baida, Opt. Lett. 32, 2942 (2007).

9. R. Gordon, D. Sinton, K. L. Kavanagh, and A. G. Brolo, Acc. Chem. Res. 41, 1049 (2008).

10. J. C. Yang, J. Ji, J. M. Hogle, and D. N. Larson, Nano Lett. 8, 2718 (2008).

11. F. Eftekhari, C. Escobedo, J. Ferreira, X. B. Duan, E. M. Girotto, A. G. Brolo, R. Gordon, and D. Sinton, Anal. Chem. 81, 4308 (2009).

12. S. Roh, T. Chung, and B. Lee, Sensors 11, 1565 (2011).

13. J. Kirk, G. Fridley, J. Chamberlain, E. Christensen, M. Hochbergb, and D. Ratner, Lab Chip 11, 1372 (2011).

14. J. A. Dionne, L. A. Sweatlock, H. A. Atwater, and A. Polman, Phys. Rev. B 73, 035407 (2006).

15. Q. Tan, A. Cosentino, M. Roussey, and H. P. Herzig, J. Opt. Soc. Am. B 28, 1711 (2011). 